\title{
Case report: Pregnancy After Vitrification of Biopsied Human Blastocysts Previously Frozen by the Slow Method
}

\author{
Batwala, M., \\ Wilding, M., \\ Create Fertility, Cheapside, London, UK \\ Dalapati, T., \\ University of Georgia, Athens, Georgia, USA \\ Nargund, G., \\ Create Fertility, Cheapside, London, UK
}

Doi: 10.19044/esj.2018.v14n27p51 URL:http://dx.doi.org/10.19044/esj.2018.v14n27p51

\begin{abstract}
Pre-implantation genetic screening (PGS) with trophectoderm biopsy is an extremely powerful technique for the determination of embryos with a high implantation potential. Patients with cryopreserved embryos seeking PGS have had limited access to this treatment due to the need to thaw, biopsy, and refreeze these embryos. This is especially true for patients with embryos cryopreserved by the slow freeze technique due to the low survival rate after thawing. In this case report, we describe the application of refreezing with the vitrification technique to embryos thawed with the slow technique and biopsied for PGS. The patient had a total of 8 blastocysts thawed, biopsied, and refrozen with vitrification. Next Generation Sequencing (NGS) of these embryos revealed that 4 blastocysts were euploid. The patients achieved a pregnancy on the the first frozen embryo transfer procedure that terminated after 11 days. The second frozen embryo transfer procedure resulted in the live birth of a $3800 \mathrm{~g}$ boy, demonstrating that thaw, biopsy, and refreeze are applicable to human reproduction even in cases where embryos have been cryopreserved by the slow technique.
\end{abstract}

Keywords: Pre-implantation genetic screening, next generation sequencing, refreezing human embryos, vitrification

\section{Introduction}

Pre-implantation Genetic Screening (PGS) with analysis of the entire chromosome content within human embryos offers patients the possibility of 
testing developing embryos for chromosome normality and a reliable method for selecting embryos for transfer (Harper, 2017). Results suggest PGS is a cost-effective procedure and decreases the time to pregnancy in patients over 37 years old (Collins et al., 2017).

Until recently, the principle technique for whole genome screening was array Comparative Genome Hybridisation (aCGH) (Harper, 2017). This technique produced results in 24 hours and allowed embryo transfer on the same cycle as embryo biopsy. However, a limitation of the technique included the necessity to perform biopsy on day 3 (with the removal of a single cell of the embryo). This caused high levels of stress for patients since results were obtained 1-2 hours before the embryo transfer procedure was scheduled with no guarantee that the embryo transfer procedure would actually take place.

Next Generation Sequencing (NGS) offers a low-cost, high-resolution method for PGS of human embryos and is considered a major breakthrough in the field of human embryology (Harper, 2017). However, the extended timeframe for NGS results (2-3 weeks) does preclude embryo transfer in the same cycle as embryo testing. Therefore embryo cryopreservation techniques are required.

Embryo cryopreservation is an established procedure since 1983 for use in human reproduction (Trounson and Mohr, 1983). Traditionally, the cryopreservation of human embryos was through the slow method where cooling occurred at $1^{\circ} \mathrm{C}$ per minute, preventing extensive intracellular crystallization (Rienzi et al., 2017). More recently, a high efficiency technique of embryo cryopreservation, termed vitrification, has been developed (Yokota et al., 2000). The efficiency of embryo survival after slow freezing compared to vitrification is known to be significantly lower $(49.7 \%$ versus $88.2 \%$, Fernandez-Gallardo et al., 2017, Rienzi et al., 2017), and the time to resumption of mitosis is delayed by up to 6 hours after slow freezing (Fernandez-Gallardo et al., 2017).

Patients wishing to have PGS with NGS on cryopreserved embryos are currently limited from accessing this technique due to the requirement of refreezing embryos. Embryo refreezing with the slow freeze technique appears not to be generally practical due to the low survival rate after thaw (FernandezGallardo et al., 2017; Rienzi et al., 2017). The vitrification of human embryos may alleviate this problem due to the reliability of this technique. Few reports have explored the use of refreezing of embryos in assisted reproduction. Mice embryos have been shown to tolerate refreezing, even after embryo biopsy (Snabes et al., 1993; Vitale et al., 1997). Mice embryos did survive the process although the authors reported several negative effects such as lower cell numbers at the blastocyst stage (Vitale et al., 1997). Human embryo refreezing has been attempted (Smith et al., 2005, Farhat et al., 2001, Kumsako et al., 2009, Ludwig et al., 1999). Human refrozen embryos have also been 
transferred to patients with successful outcomes (Farhat et al., 2001, Safari et al., 2016). Despite this, refreezing of human embryos is not a widely used procedure in the IVF lab at the present time.

In this case report, we describe the use of vitrification for the refreezing of embryos previously frozen with the slow technique. This report demonstrates that slow-thawed and biopsied embryos can be refrozen with vitrification with no loss of potential, and ongoing clinical pregnancies can be obtained with this technique. The data demonstrate that patients with embryos cryopreserved by the slow method should not be excluded from NGS procedures for this reason alone.

\section{Case Report}

The patients in the present report had a history of trying to conceive for 4 years. The female patient was 36 years old at the time of treatment, and the male was 41 years old. The couple was diagnosed with idiopathic (unexplained) infertility and had completed one long protocol cycle of IVF at a different treatment unit. In this unit, she had 29 eggs collected, of which 20 were mature and 18 fertilised. This resulted in 11 blastocysts, of which 1 embryo was transferred fresh and 10 embryos were frozen by the slow method (Edgar et al., 2017). The fresh embryo transfer resulted in a pregnancy which ended in a miscarriage at 10 weeks of gestation. The couple subsequently attempted 2 further cycles of single embryo transfer (SET) at the same centre without success. The couple transferred their remaining 8 frozen embryos to CREATE Fertility for analysis with PGS using NGS. The 8 embryos were thawed according to the manufacturer's instructions by briefly plunging the closed straws for 1 minute into water warmed to $30^{\circ} \mathrm{C}$, emptying of the contents into a petri dish (NUNC, Rochester, USA), and transferring the embryos into sequential thawing solutions (SAGE, San Clemente, USA). Once thaw was completed, embryos were transferred to culture medium (GTL, Vitrolife, Gothenburg, Sweden) for 2 hours to enable the blastocysts to recover and re-expand before biopsy.

Once recovery and blastocyst re-expansion were complete, a small portion of the trophectoderm of the embryo was removed with standard trophectoderm biopsy techniques (McArthur et al., 2005). Trophectoderm samples were washed and placed into a PCR tube (Eppendorf, Hamburg, Germany) in a solution of lysis buffer (Reprogenetics, Oxford, UK). Blastocysts were immediately refrozen using the Kitazato vitrification technique with the Cryotop loading device (Kitazato, Tokyo, Japan). The samples were sent to the genetics lab (Reprogenetics, Oxford, UK) to be analysed with NGS (Illumina, USA). The analysis revealed 4/8 of the embryos were chromosomally normal (Table I). Abnormalities of the other 4 embryos appeared to be multiple aneuploidies (Table I). 
Table I. Results after NGS.

\begin{tabular}{|c|c|l|l|c|}
\hline $\begin{array}{c}\text { Grade of } \\
\text { Frozen } \\
\text { Blastocyst }\end{array}$ & $\begin{array}{c}\text { Grade of blastocyst at } \\
1 \text { st slow thaw }\end{array}$ & $\begin{array}{c}\text { Result of NGS } \\
\text { Biopsy }\end{array}$ & $\begin{array}{c}\text { Grade of Embryo } \\
\text { prior to transfer }\end{array}$ & Embryo Outcome \\
\hline $3 \mathrm{Bb}$ & $3 \mathrm{Bb}$ & Euploid & $5 \mathrm{Ab}$ & $\begin{array}{c}\text { 1st DET-Biochemical } \\
\text { Pregnancy }\end{array}$ \\
\hline $3 \mathrm{Bc}$ & $3 \mathrm{Bc}$ & Euploid & $4 \mathrm{Bb}$ & - \\
\hline $3 \mathrm{Bb}$ & $3 \mathrm{Bb}$ & $\begin{array}{l}\text { Complex } \\
\text { Aneuploidy }\end{array}$ & N/A-discarded & - \\
\hline $3 \mathrm{Ab}$ & $3 \mathrm{Ab}$ & $\begin{array}{l}\text { Complex } \\
\text { Aneuploidy }\end{array}$ & N/A-discarded & - \\
\hline $4 \mathrm{Bb}$ & $4 \mathrm{Bb}$ & $\begin{array}{l}\text { Complex } \\
\text { Aneuploidy }\end{array}$ & N/A-discarded & - \\
\hline $4 \mathrm{Bb}$ & $4 \mathrm{Bb}$ & $\begin{array}{l}\text { Complex } \\
\text { Aneuploidy }\end{array}$ & N/A-discarded & - \\
\hline $4 \mathrm{Cb}$ & $4 \mathrm{Cb}$ & Euploid & $3 \mathrm{Ab}$ & $\begin{array}{l}\text { 2nd DET, singleton } \\
\text { live birth } 41 \text { weeks } \\
\text { gestation. }\end{array}$ \\
\hline $5 \mathrm{Ab}$ & $5 \mathrm{Ab}$ & Euploid & $4 \mathrm{Bb}$ & \\
\hline
\end{tabular}

The couple was prepared for frozen embryo transfer with oral oestrogens ( $2 \mathrm{mg}$ Progynova, Bayer, Switzerland 4 times a day) from day 2 of the menstrual cycle. When the endometrium reached a minimum of $7 \mathrm{~mm}$ thickness and a distinctive triple layer was observed, vaginal progesterone was administered (Cyclogest, Actavis, UK 800mg/day).

Five days after the start of progesterone administration, two normoploid blastocysts grade $5 \mathrm{Ab}$ and $4 \mathrm{Bb}$ (Gardner et al., 2000) were thawed by the warming technique (Kitazato). The patient requested a Double Embryo Transfer (DET). CREATE Fertility Unit's policy is to actively encourage Single Embryo Transfer (SET) when euploidy is confirmed. The 2 embryos survived the warming process and leading to a biochemical pregnancy 9 days after transfer, confirmed by serum beta Human Chorionic Gonadotrophin (bhCG). However, this pregnancy ended 2 days later, which was diagnosed by declining serum beta human Chorionic Gonadotrophin (bhCG) levels.

The second frozen embryo transfer was prepared using the same method, i.e., use of oral oestrogens to prepare the endometrium. Once the uterus had reached $7 \mathrm{~mm}$ and a distinctive triple layer was observed, the luteal phase was triggered with progesterone. Five days later, the remaining 2 euploid blastocysts $3 \mathrm{Ab}$ and $4 \mathrm{Bb}$ were thawed and transferred. The couple achieved a live birth of a $3800 \mathrm{~g}$ male baby at 41 weeks gestation.

\section{Discussion}

The need to refreeze human embryos has been one of the risks of assisted reproduction procedures, for example, if the procedure must be abandoned during frozen embryo transfer procedures. With the slow freeze technique, the low efficiency of survival after thaw indicates that refreezing is an extremely risky procedure and can lead to failure of survival of the embryo 
after the second thaw process. However, with the introduction of the high efficiency cryopreservation procedure of vitrification, the refreezing of human embryos has become a distinct possibility. The need to refreeze human embryos could become significant with the introduction of NGS as an established PGS technique because patients with cryopreserved embryos may wish to have PGS, which would require thawing of their embryos, biopsy of suitable blastocysts, and refreezing of the biopsied blastocysts prior to analysis with NGS. Embryo refreezing permits the field of assisted reproductive technology to adopt a strategy that enables NGS on previously cryopreserved embryos to allow this powerful technology to be available to all patient groups.

A few reports have described the procedure of embryo refreezing by vitrification (Farhat et al., 2001, Safari et al., 2016). Theoretically vitrification and slow freezing both rely on dehydration of human cells and are therefore not incompatible, no reports have explored the refreezing by vitrification of human blastocysts previously frozen by the slow freeze method. In this report, we outline a successful pregnancy after thawing of human blastocysts frozen by the slow freeze method, trophectoderm biopsy for PGS with NGS, and same day refreezing with vitrification.

The fact that embryos can tolerate this process and retain their capacity for implantation, and establish pregnancy suggests that PGS with NGS can be offered to patients with previously cryopreserved embryos even in cases where these are cryopreserved with the slow freeze method.

\section{Acknowledgements}

We thank the patients concerned who very generously agreed for use to use their clinical data in this publication.

\section{References:}

1. Collins, SC, Xu, X, Mak, W. (2017). Cost-effectiveness of preimplantation genetic screening for women older than 37 undergoing in-vitro fertilisation. J Assist Reproduct Genet 34; 1515-1522..

2. Edgar DH, Archer J, Gook DA. (2017). Slow Freezing and Thawing of Human Cleavage Stage Embryos. Methods Mol Biol; 1568:119129.

3. Farhat M, Zentner BS, Lossos F, Bdolh Y, Holtzer H, Hurwitz. (2001). A Successful pregnancy following replacement of embryos previously refrozen at blastocyst stage: case report. Hum Reprod;16, 337-339.

4. Fernandez-Gallardo E, Spiessens C, D’Hooghe T, Debrock, S. (2017). Effect of day 3 morphometrics and morphometrics on survival and implantation after slow-freezing-thawing and after vitrificationwarming: a retrospective cohort study. Reprod Biol Endocrinol.;15, 79. 
5. Gardner DK, Lane M, Stevens J, Schlenker T, Schoolcraft WB. (2000). Blastocyst score affects implantation and pregnancy outcome: towards a single blastocyst transfer. Fertil Steril.;73:1155-8.

6. Harper J. Preimplantation genetic screening. (2018). J Med Screen25:1-5..

7. Kumasako Y, Otsu E, Utsonomiya T, Araki Y. (2009). The efficacy of the transfer of twice frozen-thawed embryos with the vitrification method. Fert Ster.;91, 383-386.

8. Ludwig M, Al-Hasani S, Felberbaum R, Diedrich K. (1999). New aspects of cryopreservation of oocytes and embryos in assisted reproduction and future perspectives. Hum Reprod.;14 Suppl 1, 162185.

9. McArthur SJ, Leigh D, Marshall JT, de Boer KA, Jansen RP. (2005). Pregnancies and live births after trophectoderm biopsy and preimplantation genetic testing of human blastocysts. Fert Ster.;84, 1628-1636.

10. Rienzi L, Gracia C, Maggiulli R, LaBarbera,AR, Kaser DJ, Ubaldi FM, Vanderpoel S, Racowsky C. (2017). Oocyte, embryo and blastocyst cryopreservation in ART: systematic review and metaanalysis comparing slow-freezing versus vitrification to produce evidence for the development of global guidance. Hum Reprod Update;23, 139-155.

11. Safari S, Faramarzi A, Agha-Rahimi A, Khalili M. (2016). Live birth in a woman with recurrent implantation failure and adenomyosis following transfer of refrozen-warmed embryos. Clin Exp Reprod Med;43; 181-184.

12. Smith LK, Roots EH, Dorsett MJ. (2005). Live birth of a healthy baby after a frozen embryo transfer with blastocysts that were frozen and thawed twice. Fert Ster;83, 198-2000.

13. Snabes MC, Cota J, Hughes MR. (1993). Cryopreserved mouse embryos can successfully survive biopsy and refreezing. J Assist Reprod Genet;10; 513-516.

14. Trounson A, Mohr L (1983). Human pregnancy following cryopreservation, thawing and transfer of an eight-cell embryo. Nature;305, 707-709.

15. Vitale NJ, Myers MW, Denniston RS, Leibo SP, Godke RA. (1997). In-vitro development of refrozen mouse embryos. Hum Rep.;12; 3103

16. Yokota Y, Sato S, Yokota M, Ishikawa Y, Makita M, Asada T, Araki Y. (2000). Successful pregnancy following blastocyst vitrification: case report. Hum Reprod.;15, 1802-1803. 